\title{
Functional characterization of Urease (ureA) in Fonsecaea pedrosoi, the causative agent of Chromoblastomycosis
}

Narla Verorrayne Gonçalo Pimentel, Fabiana Alves Silva Brandão, Raffael Júnio Araújo de Castro, Camille Silva Florencio, Maria Sueli Soares Felipe, Janice Lisboa Demarco, Anamélia Lorenzetti Bocca, Larissa

Fernandes Matos* Presenting author: larissaf@unb.br

Faculty of Ceilandia; Department of Cell Biology, Institute of Biological Sciences, University of Brasília-DF

\begin{abstract}
Fonsecaea pedrosoi, the main agent that causes chromoblastomycosis (CBM) in humans, is a filamentous fungus with a worldwide distribution. CBM is prevalent in tropical and subtropical regions, with high incidence in Africa and Latin America. The biology, virulence factors and mechanisms of pathogenesis of $F$. pedrosoi are still poor understood and for this reason studies involving the mechanisms this fungal pathogen uses to stablish the infection are essential for the improving the knowledge about the disease and potentially develop new antifungal therapies. Recently our group described two genetic transformation methods to facilitate the gene function studies in this fungus. We use biolistic transformation to knock out $F$. pedrosoi ureA gene (coding for Urease) by homologous recombination and gene replacement. The main objective of this work was to characterize $F$. pedrosoi urease and evaluate its potential role on virulence of this fungal pathogen.
\end{abstract}

\section{Methodology}

Cultivation of F. pedrosoi on Potato Dextrose Broth for 7 days $/ 28^{\circ} \mathrm{C}$

Separation of the conidia

Biolistic transformation of cassette deletion

Selection for HygR transformants

Screening on Christensen's Urea Agar for urease negative transformants

Genomic DNA extraction

PCR to confirm HygR integration on the urease locus

Phenotypic characterization of the knock out mutants

\section{Results}

This work describes, for the first time, the literature to date, the construction and obtaining of a $F$. pedrosoi mutant by homologous recombination and gene deletion. The gene encoding the enzyme urease is well characterized in other fungi such as Cryptococcus sp. and Coccidioides posadasii as a factor of fungal virulence. In $F$. pedrosoi the absence of the ureA gene was confirmed by the inability of the mutant $\Delta$ ureA to produce urease in Christensen's Urea Agar. The mutant was able to grow in culture medium with different nitrogen sources but showed a growth defect when urea was the only source of nitrogen. No growth defects were observed on the stress conditions evaluated (data not shown). In vitro virulence experiments are underway to date (data not shown).

A
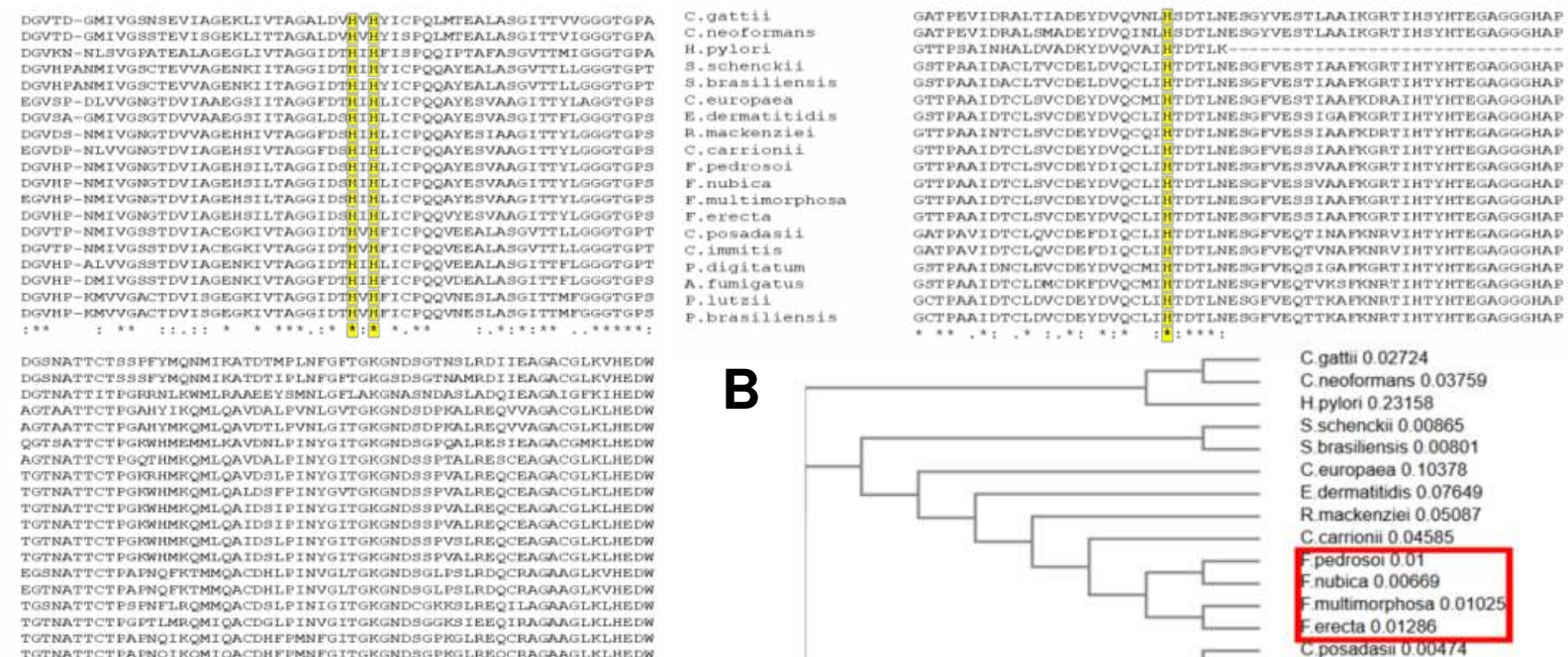

B

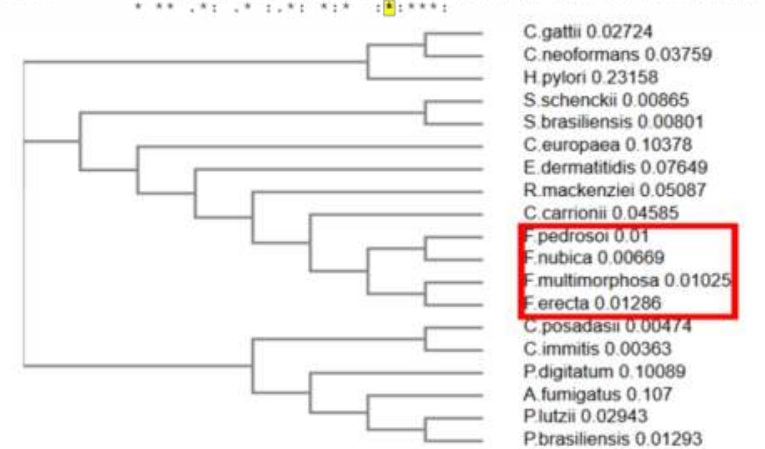

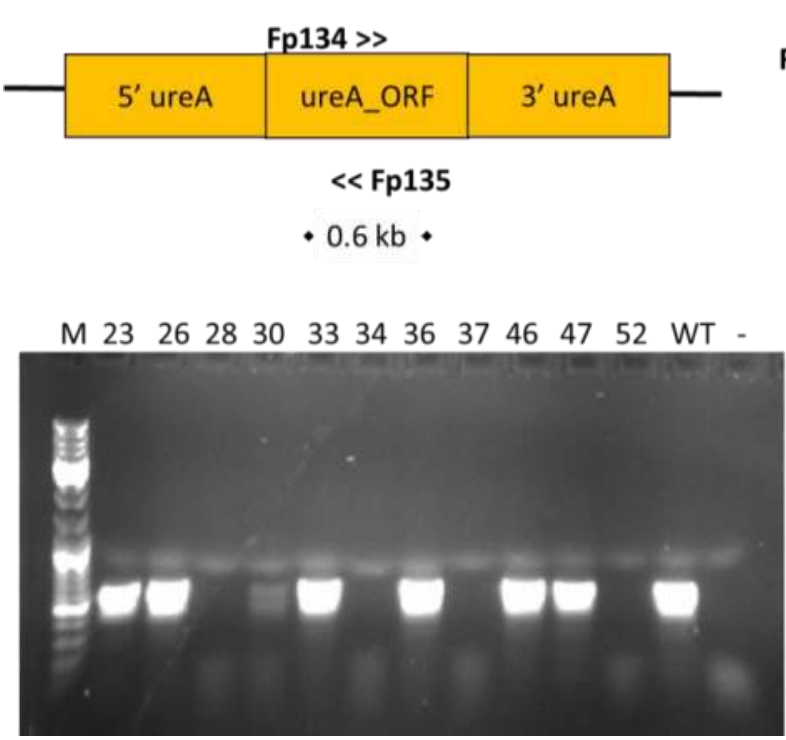

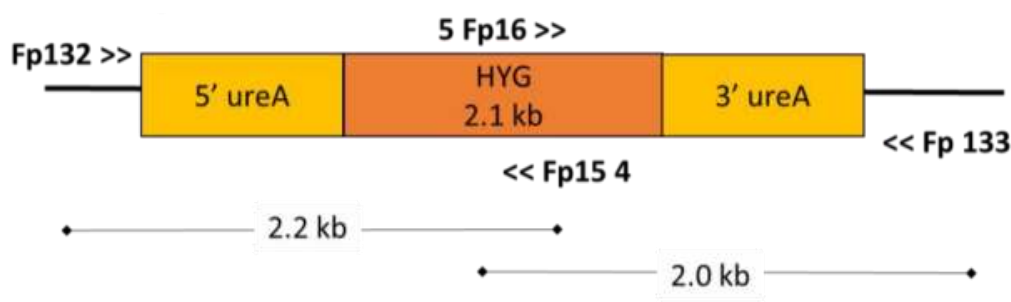

Figure 2- Confirmation of urease deletion in $F$. pedrosoi, the deleted mutants are indicated on the figure as well as the expected size of the fragments. The controls were genomic DNA of CBS271.37 and $\mathrm{H}_{2} \mathrm{O}$

A
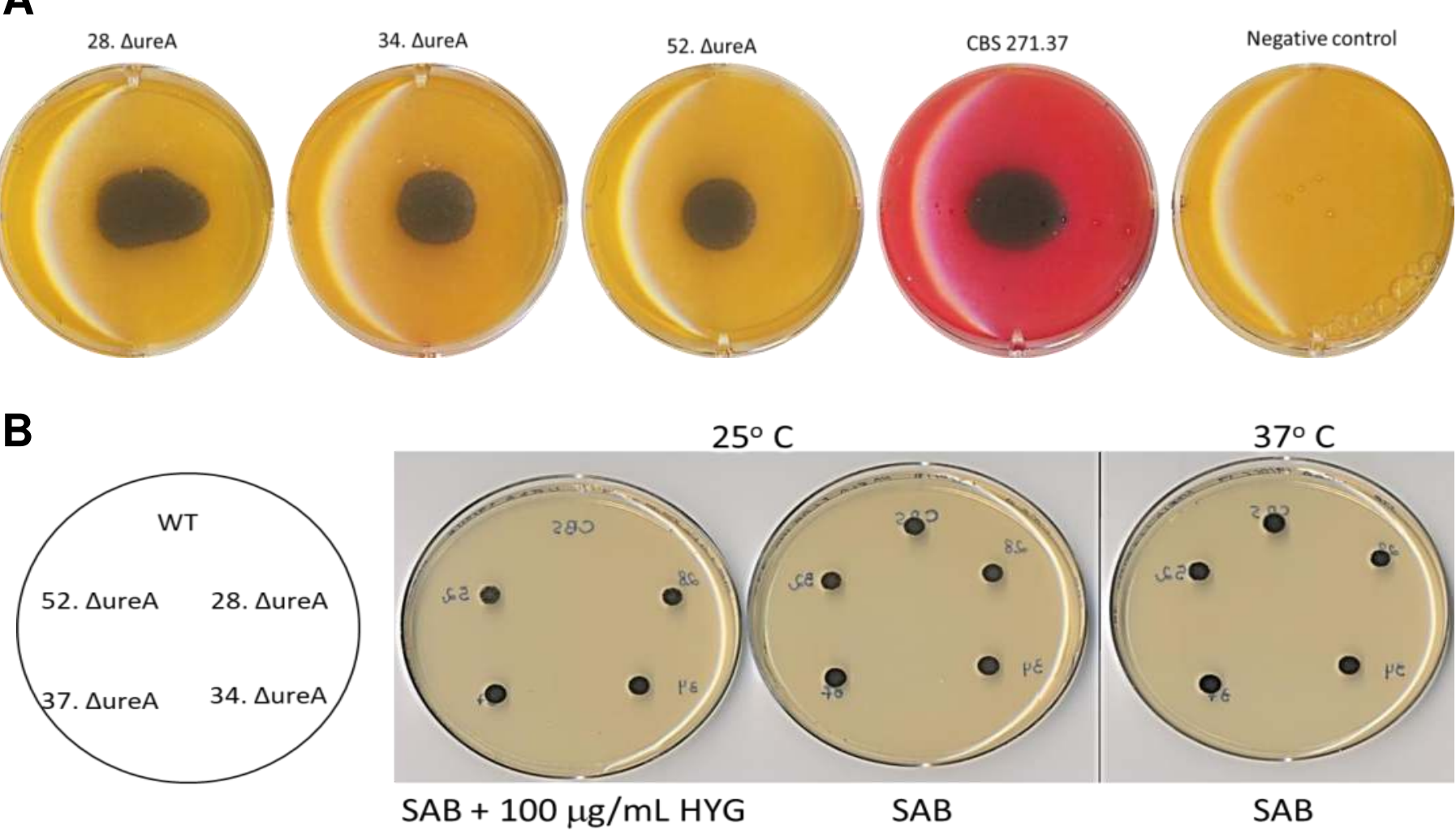

C

\section{DureA}
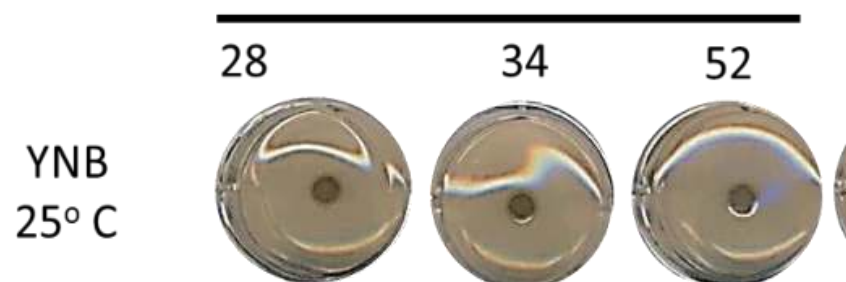

CBS

YNB

$37^{\circ} \mathrm{C}$

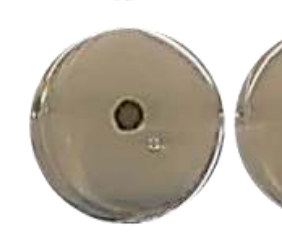

0

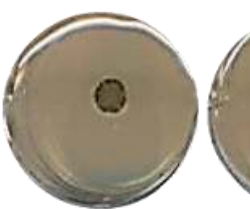

YNB
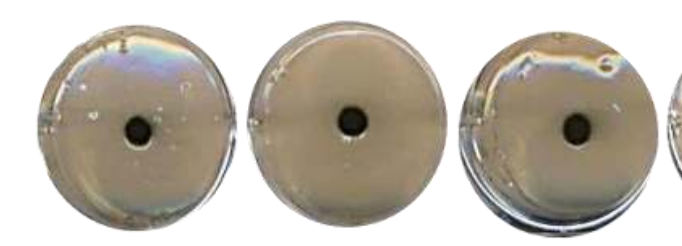

Figure 3- Urease mutant phenotypes of F. pedrosoi after growth on activity detection on Christensen's Urea Agar, negative urease producers do not change the yellow to pink color of the medium. (B) Evaluation of sensitivity to high temperature growth on Sabouraud agar plates. (C) Evaluation of the ability of $F$. pedrosoi urease mutants to use different nitrogen sources on $\mathrm{NH}_{4} \mathrm{SO}_{4}, 30 \mathrm{mM}$ L-glutamine, 30 $\mathrm{mM}$ urea, $5 \mathrm{mM} \mathrm{L}$-arginine. All plates were photographed after 7-10 days of growth at indicated temperatures.

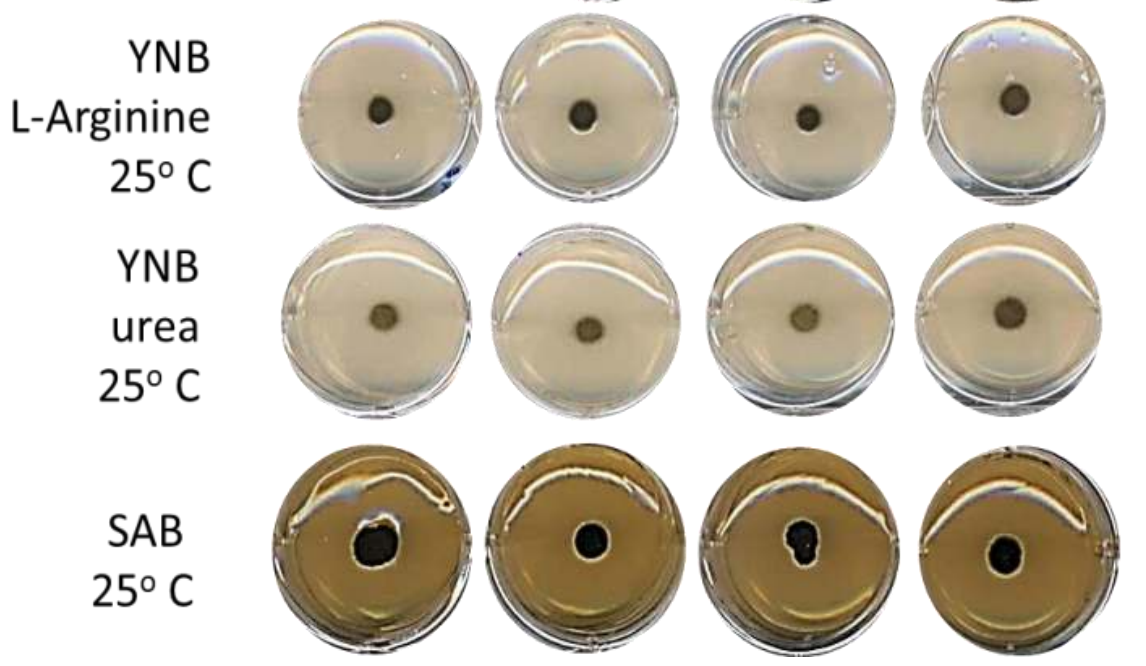

Conclusion

For the first time in literature to date, a knock out mutant was constructed in F. pedrosoi, which is one of the main causative agent of CBM. The $\triangle$ ureA is under phenotypic characterization and the data generated by this work will potential identify the role of urease on $F$. pedrosoi virulence traits. This work opens new perspectives for fungal pathogenesis studies by gene deletion in this organism.

\section{Financial support}

